\title{
Les transferts de TPE d'un milieu urbain vers un milieu rural isolé : des trajectoires spatiales atypiques riches d'enseignements sur la coévolution des TPE et des réseaux de leurs dirigeants
}

\section{Relocations of small businesses from urban to rural areas : atypical spatial trajectories bringing new insights into the coevolution of small businesses and their founders' networks}

\author{
Séverine Saleilles ${ }^{\mathrm{a}^{*}}$ et Bertrand Sergot ${ }^{\mathrm{b}}$ \\ ${ }^{*}$ a Maître de Conférences, COACTIS, Université de Lyon \\ ${ }^{b}$ Maître de Conférences, PESOR, Université Paris-Sud
}

\section{Résumé}

Les transferts de TPE d'un milieu urbain vers un milieu rural isolé constituent un type de trajectoire spatiale particulièrement atypique au regard de la tendance lourde à l'inertie spatiale des entreprises de petite taille, soulignée de longue date, et par les données statistiques existantes, et par la littérature académique. À partir d'une analyse croisée des travaux de recherche existants et des données empiriques issues de l'étude de 10 cas, nous proposons une typologie des logiques de départ de ces entrepreneurs et des affaires qu'ils dirigent hors de leur milieu d'origine. Nous dégageons ensuite les axes de réflexion les plus prometteurs, au regard de nos données empiriques en vue d'améliorer de la compréhension des motivations et des conséquences de ces trajectoires spatiales particulières

*Adresse email : severine.saleilles@univ-st-etienne.fr doi:10.3166/ges.15.89-115 @ 2013 Lavoisier, Paris. Tous droits réservés. 
mais également de concevoir des dispositifs d'accompagnement à même d'en faciliter la réalisation afin que les entrepreneurs et les territoires ruraux susceptibles de les accueillir puissent trouver intérêt à ce type de parcours entrepreneurial.

(c) 2013 Lavoisier, Paris. Tous droits réservés.

\section{Summary}

The relocation of a small business from an urban to a rural area is an atypical spatial trajectory considering the well-documented tendency towards spatial inertia displayed by small businesses. Based on an extended literature review and 10 case studies, we propose a typology of the relocation logics of relocating entrepreneurs and the businesses they run. We then identify the ways in which our empirical work contributes to a better understanding of the motivations and consequences of such trajectories. Finally, we formulate some proposals to improve the design of schemes to support this type of geographical moves and to attract relocating small businesses to specific rural areas.

(C) 2013 Lavoisier, Paris. Tous droits réservés.

Mots clés : Transfert, Mobilité, TPE, réseaux, migrations ville-campagne.

Keywords : Relocation, mobility, small business, networks, urban-rural migration.

\section{Introduction}

En France, les données statistiques disponibles indiquent que 1,8 million de personnes, dont plus de 800000 actifs, ont quitté un pôle urbain pour la campagne entre 1990 et 1999 (Observatoire des territoires, 2005). Les résultats des enquêtes annuelles de recensement de 2004 et 2005 montrent que ce retournement démographique touche, de plus en plus, les espaces ruraux les plus isolés (Morel et Redor, 2006). Deux enquêtes effectuées en 1999 et en 2004 auprès des maires de petites communes rurales par Mairie-Conseils, service de la Caisse des Dépôts et Consignations, indiquent en outre qu'environ $5 \%$ des nouveaux installés dans ces communes sont des créateurs ou des repreneurs d'entreprises. À partir du recensement de 1999, Sencébé et Lepicier (2007) indiquent pour leur part que 16401 artisans, commerçants et chefs d'entreprise ont migré vers un espace rural isolé. Une partie non-précisée mais significative de ces entrepreneurs disposait à l'origine d'une affaire. Dans la foulée de leur déménagement, ils ont procédé au transfert de cette affaire.

À l'instar de l'INSEE, nous définissons un transfert comme « une opération par laquelle une entreprise déplace tout ou partie de ses éléments et moyens de production d'un lieu (lieu de départ) vers un autre lieu (lieu d'arrivée) » (INSEE, 2011, p. 22). Parmi les multiples modalités possibles de mise en œuvre de transferts par des entreprises, nous avons choisi de concentrer notre attention sur ce que l'INSEE qualifie de transferts complets, c'est-à-dire sur les déplacements géographiques d'entreprises «qui s'accompagnent de la disparition totale de l'activité au lieu de départ et [dans le cadre desquels] il n'y avait aucune activité préexistante sur le lieu d'arrivée », réalisés par des entrepreneurs entre leur milieu urbain d'origine et une zone rurale isolée dans laquelle ils ne sont pas nés et qui est située dans une autre région ou un autre pays que leur commune urbaine de provenance ${ }^{1}$. Conformément à

\footnotetext{
${ }^{1}$ Dans la suite de l'article, le terme de transfert fait référence à cette catégorie particulière de transferts complets.
} 
la typologie des espaces ruraux proposée par l'INRA et l'INSEE (Hilal et Schmitt, 1997; Nicot, 2005), l'expression de rural isolé désigne, dans cet article, l'ensemble des communes françaises dont l'éloignement ne permet pas à leurs résidants des déplacements domiciletravail quotidiens avec une aire urbaine ${ }^{2}$.

Ces choix sont dictés par des considérations d'ordre pratiques, théoriques et méthodologiques.

D'un point de vue pratique, ces nouveaux arrivants transférant leur entreprise sont susceptibles de jouer un rôle crucial dans le développement économique et la revitalisation des zones rurales isolées dans lesquelles ils s'installent (Chevalier, 2000), au travers des emplois qu'ils y créent, mais également par le renouvellement et la diversification qu'ils peuvent apporter au tissu économique local (Font, 2000). Par ailleurs, un transfert d'activités vers le milieu rural constitue, pour le dirigeant qui le met en œuvre, un changement d'autant plus radical et complexe à piloter qu'il coïncide avec son installation - et celle de sa famille - dans un environnement relativement méconnu. La mise à jour des logiques sous-jacentes à ce type de démarche et des motivations des entrepreneurs concernés constitue donc un prérequis essentiel à la compréhension de leurs conditions de réussite et, par là même, à toute réflexion visant à concevoir des dispositifs d'accompagnement à même de favoriser cette réussite.

D'un point de vue théorique, ce type de trajectoire spatiale apparaît particulièrement atypique au regard des enseignements de la littérature académique. Les travaux de recherche effectués sur le sujet, tant en sciences de gestion qu'en géographie, ou encore dans le champ des sciences économiques ou de la sociologie, convergent en effet vers un constat lourd : la tendance particulièrement prononcée des entrepreneurs à créer leur entreprise dans l'environnement local qui leur est le plus familier, à savoir celui dans lequel ils résidaient et travaillaient au préalable, et à y demeurer ensuite durablement. Des cadres conceptuels solides et largement validés empiriquement ont été construits afin d'expliquer la prégnance de ces comportements de localisation inertiels. Ils s'appuient en particulier sur le rôle crucial de l'activation, par l'entrepreneur, de son réseau social préexistant pour la mobilisation des ressources nécessaires à la création puis au développement de son entreprise. L'existence même de transferts de Très Petites Entreprises soulève des interrogations de nature à justifier la réalisation de travaux de recherche visant à mieux comprendre les logiques conduisant certains entrepreneurs à adopter ce type particulier de trajectoire géographique et les conséquences qu'un tel choix peut avoir tant pour leur affaire que pour leur nouveau territoire d'accueil.

D'un point de vue méthodologique, opter pour l'étude d'un type de trajectoire spatiale bien particulier nous est apparu d'autant plus riche que cette option est susceptible, à partir de l'étude d'un nombre de cas relativement restreint mais dont les caractéristiques sont homogènes, de favoriser la mise à jour des principaux questionnements et pistes de recherche ouverts par le phénomène du transfert de TPE (Yin, 2003).

Le présent article s'articule autour de la question de recherche suivante : Si les forces qui poussent à la création et au maintien des nouvelles entreprises dans les environne-

\footnotetext{
${ }^{2}$ Statistiquement, est considérée comme appartenant au rural isolé toute commune dont moins de $20 \%$ des actifs travaillent dans un pôle urbain offrant 2000 emplois ou plus (Hilal et Schmitt, 1997).
} 
ments urbains familiers à leurs fondateurs sont si puissantes, comment expliquer que certains d'entre eux, après s'être conformés au modèle général, choisissent d'adopter une trajectoire apparemment déviante? Au sein de ce questionnement général, nous avons porté une attention particulière à la compréhension du rôle que jouent les réseaux de l'entrepreneur dans ces décisions afin de mettre à jour les enrichissements et les éventuelles remises en question que l'étude de choix aussi atypiques est susceptible d'apporter aux cadres d'analyse de l'évolution des réseaux du dirigeant de TPE.

La démarche que nous avons adoptée est de nature exploratoire. Elle s'appuie conjointement sur l'analyse de la littérature et sur l'exploitation d'entretiens réalisés avec 10 dirigeants ayant transféré leur TPE en milieu rural isolé. Dans un premier temps, les grands cadres conceptuels utilisés sont exposés et les définitions retenues pour les notions mobilisées sont explicitées et justifiées. Ensuite, nous présentons les principaux choix méthodologiques qui ont guidé la collecte et le traitement des données empiriques ainsi qu'un rapide exposé des caractéristiques des 10 cas étudiés. Dans une troisième partie, nous présentons les résultats issus de cette étude exploratoire. Enfin nous exposons dans une dernière partie les implications et prolongements possibles de notre travail.

\section{Le transfert de TPE vers un milieu rural : une trajectoire atypique et encore peu étudiée}

Le transfert d'un milieu urbain vers un milieu rural constitue un phénomène atypique au regard de la tendance lourde à l'inertie des TPE (1.1.). Son analyse peut permettre d'enrichir et/ou de questionner les modèles de coévolution de la TPE et des réseaux de son dirigeant (1.2.) dans la mesure où la reconfiguration des réseaux est une question centrale dans cette forme d'entrepreneuriat néo-rural (1.3.).

\subsection{L'inertie spatiale des TPE}

De manière générale, les données statistiques disponibles indiquent que les entreprises et leurs établissements sont des entités peu mobiles géographiquement (Van Dijk et Pellenbarg, 2000; Jourdan, 2004). De nombreux travaux académiques ont été réalisés afin de mieux comprendre les phénomènes sous-jacents à ces constats chiffrés récurrents.

Il apparaît tout d'abord que la plupart des entrepreneurs créent là où ils vivent, et ce aussi bien pour les créations de petites entreprises de type artisanal ou commercial que pour celles d'entreprises de haute technologie. La prédominance de ce type de choix de localisation s'explique par la conjonction de plusieurs logiques convergeant lors du processus de démarrage d'entreprises nouvelles :

- l'utilisation, par l'entrepreneur, de son réseau personnel afin de découvrir de nouvelles opportunités de marché et d'initier le processus de mobilisation des ressources qu'implique la démarche entrepreneuriale (Sorenson et Audia, 2000; Figuereido et al., 2002; Sorenson et Stuart, 2003);

- la volonté des entrepreneurs, au-delà de l'instrumentalisation à des fins économiques de leur réseau personnel, de manifester leur attachement identitaire à leur région d'origine (Huault, 2004; Reix, 2008);

- enfin, de manière plus prosaïque, la nécessité de concilier les impératifs de la 
démarche entrepreneuriale avec une vie privée ou familiale (relativement) équilibrée, notamment en évitant d'être contraints à déménager dans un nouveau logement (Sorenson et Audia, 2000; Chanteau, 2001; Sergot, 2007).

Après la création, même lorsque la localisation du siège social de l'entreprise change, ces transferts se font, dans leur immense majorité, à l'échelle locale (Jourdan; 2004; Stam, 2007). La mobilité lointaine, qui recouvre l'ensemble des transferts d'entreprises réalisés entre des régions ou des pays différents (Romo et Schwartz, 1995; Van Dijk et al., 2000 ; Jourdan, 2004), constitue donc encore aujourd'hui une exception remarquable dans les comportements organisationnels. La première explication avancée dans la littérature pour rendre compte de cette inertie spatiale forte et croissante avec l'âge de l'entreprise est l'existence de coûts de transfert non-triviaux (Brouwer et al., 2004) qui se matérialisent sous la forme de coûts irrécouvrables (sunk costs, Williamson, 1986; Zimmermann, 1995) que les entreprises doivent supporter en relation avec leurs investissements dans des actifs localisés et peu mobiles (actifs place-specific, Williamson, 1986). Ces investissements qui attachent l'entreprise au lieu d'implantation qu'elle a initialement choisie sont essentiellement de trois ordres :

- des investissements en actifs physiques (terrains, bâtiments, équipements) spécifiques à l'activité de l'entreprise et qui ne peuvent être déplacés sans difficultés ni coûts ;

- des investissements en ressources humaines, le personnel recruté localement possédant parfois des compétences très spécifiques ou acquérant au fil du temps des savoirfaire propres au métier de l'entreprise qui peuvent se perdre lorsque les salariés en question refusent de suivre le transfert de leurs postes de travail (Zimmermann, 1995 ; Sergot, 2007);

- la construction de relations de confiance avec les clients et les partenaires de l'entreprise au travers de contacts répétés en face-à-face requérant une proximité géographique forte. La proximité physique avec les clients représente une source d'avantages concurrentiels d'autant plus importante que l'entreprise est petite et sa gestion personnalisée (Torrés, 2003; Stam, 2007).

C'est sur cette dernière catégorie d'investissements, ceux liés aux réseaux dans lesquels est insérée la TPE, que nous souhaitons porter une attention particulière dans le cadre de cet article., La littérature montre en effet que la TPE et le réseau de son dirigeant co-évoluent.

\subsection{La co-évolution de la TPE et des réseaux de son dirigeant}

Le rôle des réseaux du dirigeant dans la création puis le développement d'une TPE a fait l'objet d'une abondante littérature. Celle-ci indique que les entrepreneurs s'appuient largement, pour démarrer leur activité, sur la mobilisation de leurs réseaux de relations interpersonnelles préexistant (Reynolds, 1991; Anderson et Jack, 2002; Hite, 2005).

La nature des réseaux mobilisés et des liens dyadiques entretenus évolue au fil du temps. Des modèles de coévolution entre le développement du réseau d'une part et le développement organisationnel d'autre part ont été développés pour en rendre compte. Butler et Hansen (1991) et Larson et Starr (1993) identifient ainsi trois phases dans l'évolution du réseau, correspondant chacune à trois phases de développement organ- 
isationnel. La première phase correspond à la création de l'entreprise : le réseau y est alors essentiellement social et de grande taille, différents liens faibles étant progressivement intégrés dans le réseau pour répondre aux besoins de l'entrepreneur. Durant la deuxième phase, dite de démarrage, la taille du réseau diminue et les liens deviennent multiplexes, combinant souvent une dimension personnelle/affective et une dimension professionnelle/économique. La troisième phase, celle de la croissance, entraîne une diversification des échanges au sein du réseau et les relations personnelles deviennent davantage inter-organisationnelles. Un modèle général d'évolution du réseau, validé par différentes études empiriques (Hill et al., 1999; Jack, 2005), se dessine alors : le réseau, d'abord à dominante sociale, se transforme peu à peu en un réseau inter-organisationnel au sein duquel l'instrumentalisation à des fins professionnelles prend progressivement le pas sur les orientations affectives, qui ne disparaissent cependant pas complétement.

Plus récemment, ce modèle général de coévolution linéaire des TPE et des réseaux mobilisés par leurs dirigeants a été amendé et complété par l'introduction de facteurs de contingence se rapportant aux caractéristiques individuelles des entrepreneurs, au secteur d'activité dans lequel ils décident de se lancer ou encore aux conditions de création de leur société (Hite et Hesterly, 2001 ; Elfring et Hulsink, 2007). La prise en compte de ces facteurs de contingence permet de mieux comprendre la diversité observée dans les parcours entrepreneuriaux, mais elle ne rend pas compte des particularités introduites dans les trajectoires des TPE par des discontinuités aussi marquantes que leur transfert, décidé par le dirigeant, d'un milieu urbain vers un milieu rural isolé.

La littérature académique insiste également sur le caractère très localisé des réseaux des dirigeants de TPE (Grossetti, 2004 ; Jack et Anderson, 2002; Schutjens et Stam, 2003). Grossetti (2004) explique cette tendance par le fait que les dirigeants créent leur affaire là où ils vivent. De plus, la proximité géographique favorise les contacts et les échanges entre acteurs et facilite ainsi l'apparition d'autres formes de proximité (Julien, 2005 ; Rallet et al., 2004) : proximité de « similitude » ou « cognitive » (partage de mêmes connaissances de base et d'expertise ou d'un même système de représentations), proximité d' «appartenance » ou « organisationnelle » (être membre ou avoir été membre d'une même organisation comme une école, une entreprise, une association, etc.), proximité « sociale» (partage de valeurs, de normes, de conventions, etc.), proximité «institutionnelle » (partage de lois et de règles du jeu institutionnel).

L'utilisation, par les entrepreneurs, de leurs réseaux localisés tendrait ainsi à les attacher à leur milieu d'origine de manière durable aussi sûrement que des liens physiques (Romo et Schwartz, 1995 ; Uzzi, 1997 ; Figuereido et al., 2002 ; Torrés, 2003 ; Stam, 2007).

Par conséquent, la décision, par un dirigeant de TPE, de transférer son entreprise d'un milieu urbain vers un milieu rural isolé constitue un choix a priori atypique à deux niveaux :

- elle implique généralement une mobilité lointaine pour l'entreprise et son dirigeant au sens où le transfert s'effectue d'une région à une autre, voire d'un pays à un autre. Ce transfert est donc systématiquement couplé à un déménagement du dirigeant; 
- elle se traduit en outre par une disjonction ${ }^{3}$ géographique forte de l'entrepreneur avec son environnement familier où se concentre la partie la plus dense et la plus riche en liens forts de son réseau (Sorenson et Audia, 2000; Grossetti, 2004) et au sein duquel il a créé son entreprise. Il se retrouve donc confronté à un nouvel environnement où il est isolé géographiquement par rapport aux autres acteurs de son champ organisationnel et dans lequel il dispose de peu ou pas de liens forts préexistants, soulevant ainsi la question du maintien de son réseau de relations interpersonnelles antérieur.

Comment expliquer, dans ces conditions, le choix de cette option de la disjonction géographique?

Pour mieux comprendre ces transferts lointains d'un milieu urbain vers un milieu rural isolé, nous pouvons mobiliser les travaux sur les migrants en milieu rural, et en particulier sur ceux qui combinent cette migration avec la création d'une entreprise.

\subsection{Le transfert de TPE vers un milieu rural : un cas atypique d'entrepreneuriat néo-rural}

Les transferts de TPE d'un milieu urbain vers un milieu rural s'inscrivent dans un courant observé dans la plupart des pays en développement : la contre-urbanisation. Ces migrations des villes vers les campagnes isolées n'obéissent pas aux critères économiques traditionnels explicatifs des phénomènes migratoires (Champion, 1989). Ce mouvement migratoire qui s'amplifie depuis les années 70 a fait l'objet de divers travaux, notamment autour d'un phénomène : l'entrepreneuriat néo-rural. Cette combinaison de la migration et de l'entrepreneuriat peut prendre trois formes : la création ex nihilo d'une entreprise, la reprise d'une entreprise et, enfin, le transfert de l'entreprise. La plupart des travaux portent sur la première forme. La littérature sur les motivations de ces migrants créateurs d'entreprises est potentiellement riche d'enseignements pour comprendre les logiques de transfert. Les motivations liées à la recherche d'une meilleure qualité de vie sont prépondérantes (Font, 2000). Ainsi, le lieu d'implantation de l'entreprise est guidée par des valeurs qualitatives (qualité de vie et beauté des paysages) ou liées à la communauté et aux possibilités de pratiquer des loisirs bien plus que par des valeurs dites économiques : fiscalité, coûts du travail, proximité de centres de recherche (Johnson et Rasker, 1995). Toutefois, cette recherche de qualité de vie passe par divers moteurs (Saleilles, 2007). Pour certains, c'est la recherche de liens (sortir de l'anonymat des villes, se rapprocher d'amis ou de la famille, etc.). Pour d'autres, c'est surtout la volonté d'un cadre de vie meilleur ou la possibilité d'accomplir un vieux rêve. Enfin, les moteurs de cette décision peuvent également être professionnels (emploi, local ou terrain disponible, opportunités à saisir, etc.). Paniagua (2002a, 2002b) montre, par exemple, que la recherche d'une qualité de vie par la migration

\footnotetext{
${ }^{3}$ Nous avons choisi d'utiliser dans cet article le terme de disjonction pour exprimer le fait que le transfert d'une TPE d'un milieu urbain vers un milieu rural isolé instaure une distance physique significative entre l'entrepreneur et son affaire d'une part, et son milieu local d'origine, d'autre part. Le terme de disjonction a été préféré à celui de découplage, parfois utilisé, car les notions de couplage et de découplage font clairement référence, dans le prolongement des travaux fondateurs du sociologue américain Harrison White, à la dimension relationnelle du couple proximité/éloignement (Granovetter, 1985, 1995) alors que le terme de disjonction se rapporte ici à la seule dimension géographique.
} 
à la campagne n'est pas liée qu'aux atouts de la vie à la campagne mais également à la possibilité d'y créer son entreprise. Pour autant, ces motivations strictement professionnelles sont surtout présentes en rural accessible, beaucoup moins en rural isolé où l'on observe surtout des logiques complexes où s'entremêlent choix personnels et familiaux, volonté entrepreneuriale et volonté de vivre dans un cadre choisi (Chevalier, 2000).

D'autres travaux s'intéressent aux spécificités des entreprises dirigées par des néo-ruraux. Ainsi, l'expérience d'un environnement différent (leur environnement urbain d'origine) permettrait aux néo-ruraux, attirés par les propriétés esthétiques et physiques du milieu rural, d'y percevoir des opportunités d'affaires non perçues par les autochtones (Anderson, 2000). Plus formés et expérimentés, les néo-ruraux créeraient des entreprises plus innovantes (North et Smallbone, 2006; Kalantaridis et Bika, 2006). Par ailleurs, les néo-ruraux utiliseraient beaucoup moins les ressources locales que les autochtones, pour leurs approvisionnements et leurs ventes, mais aussi et surtout comme sources d'informations sur les opportunités de marché. Pour autant, les interactions avec leur réseau informationnel seraient, malgré la distance, plus fréquentes chez les néo-ruraux; ces derniers seraient donc « encastrés » dans un contexte qui n'est pas territorial mais fonctionnel (Kalantaridis et Bika, 2006). Toutefois, les travaux existants ne distinguent pas les diverses formes d'entrepreneuriat néo-rural (création, reprise ou transfert) dont sont issues ces entreprises.

Or, le transfert de TPE mérite un éclairage spécifique. En effet, dans les deux autres cas (création et reprise), l'entrepreneur néo-rural migre en milieu rural et y créé/reprend ensuite une entreprise. Il peut donc concevoir son projet entrepreneurial en fonction de son projet de vie et des caractéristiques de son nouveau milieu de vie. Comme tout créateur, il doit se constituer un réseau d'affaires mais dans un contexte particulier, celui d'une migration, qui implique donc un réseau social dans son milieu d'accueil faible et des liens mobilisables dans son milieu urbain d'origine (Saleilles, 2007). En revanche, quand il y a transfert, l'entreprise préexiste à la migration, et la plupart du temps à la décision de migrer. Donc, le mode de fonctionnement de la TPE ne correspond pas nécessairement au nouveau projet de vie de son dirigeant, ni à son nouveau milieu. La question à laquelle est confrontée le dirigeant qui transfère sa TPE n'est donc pas celle de la constitution d'un réseau d'affaires (comme dans les autres cas d'entrepreneuriat néo-rural), mais bien celle de la reconfiguration d'un réseau d'affaires existant pour l'adapter au nouveau projet entrepreneurial et de vie (les deux étant entremêlés) et au nouveau milieu dans lequel ces projets se réalisent. Si l'on considère que réseau et TPE co-évoluent, il s'agit également de l'adaptation de la TPE aux possibles évolutions du réseau d'affaires suite au transfert.

Les apports des travaux sur les migrants créateurs ne sont donc pas systématiquement transposables au cas des migrants qui transfèrent leur TPE. C'est la raison pour laquelle nous avons choisi de compléter les travaux existants en menant une étude empirique spécifiquement consacrée à ce type particulier de démarche.

\section{Méthodologie de l'étude}

Afin de mieux comprendre la problématique du transfert de TPE d'un milieu urbain vers un milieu rural isolé, nous avons retenu 10 cas de dirigeants ayant réalisé cette démarche. L'INSEE (2011) ne considère comme des transferts que les cas où une entreprise a changé de lieu d'implantation sans changer de numéro SIREN, donc les cas présentant une conti- 
nuité administrative de l'exploitation. Cette approche nous paraît trop restrictive au regard des définitions utilisées dans la littérature académique. Nous avons donc opté pour une définition plus large de la notion de transfert de TPE d'un milieu urbain vers une zone rurale isolée en y intégrant toutes les situations où le dirigeant d'une entreprise créée en milieu urbain a décidé de procéder simultanément au déménagement de son lieu de résidence et de son lieu habituel de travail vers une zone rurale isolée, tout en conservant au moins une partie de sa clientèle et de son activité antérieure. Cette définition inclut donc, à la différence de celle de l'INSEE, les cas où la dissolution administrative des entreprises dans le milieu de départ des entrepreneurs (milieu urbain situé en France ou à l'étranger) est suivie d'une réactivation de l'entreprise dans leur milieu rural d'accueil.

Les principales caractéristiques des cas étudiés sont présentées dans le tableau 1. Ces cas sont issus d'une recherche plus large réalisée sur deux terrains d'étude (zones classées « rural isolé » des départements du Gers et de l'Ardèche voir carte 1) et portant sur l'entrepreneuriat néo-rural sous ses différentes formes : transferts d'entreprises mais aussi créations ex nihilo et reprises.

Carte 1 : Départements d'implantation des entrepreneurs interrogés

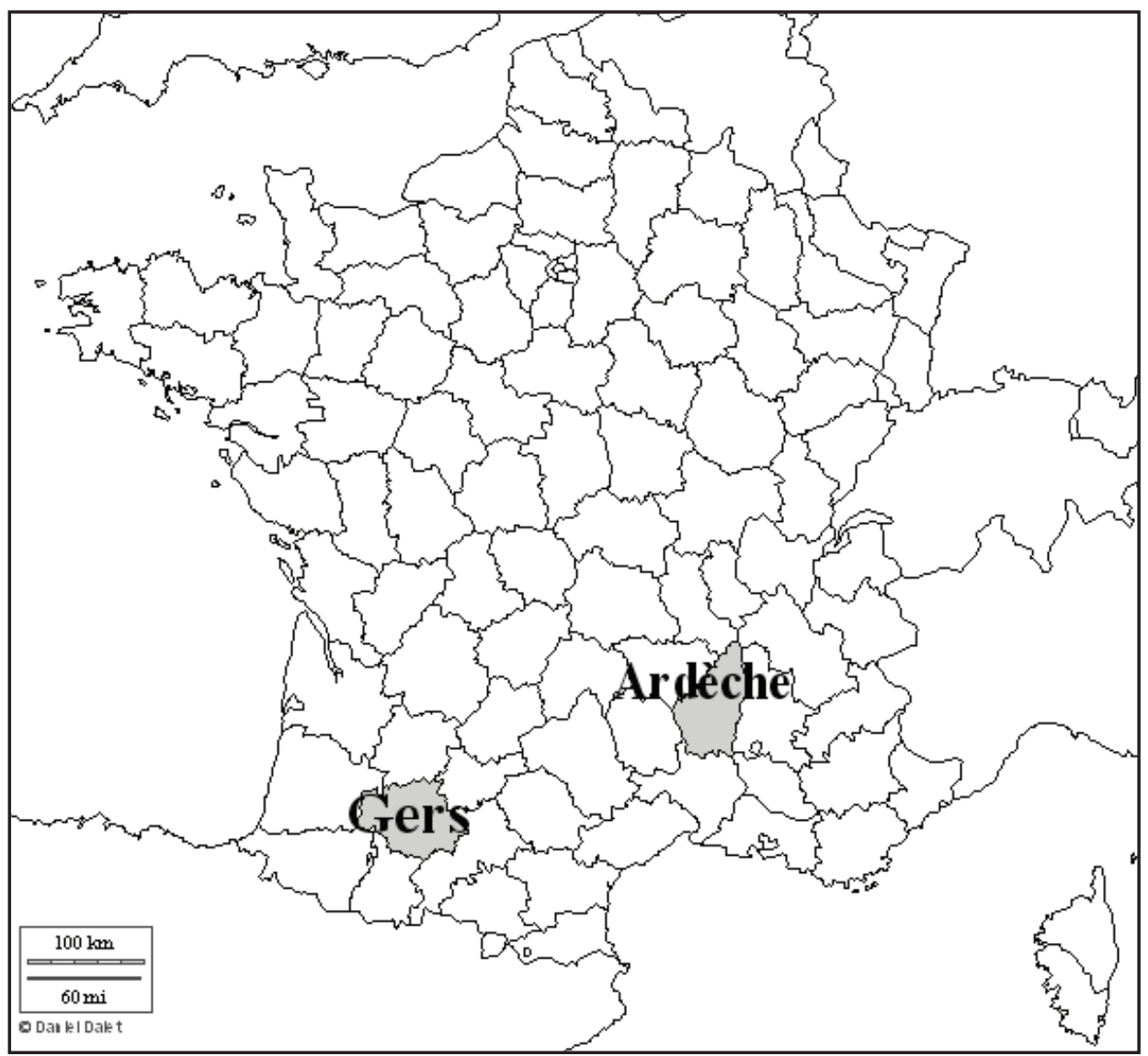


Tableau 1 : Caractéristiques des enquêtés

\begin{tabular}{|c|c|c|c|c|}
\hline Enquêtés & $\begin{array}{l}\text { Date et lieu création } \\
\text { Date et lieu transfert }\end{array}$ & $\begin{array}{c}\text { Age et situation } \\
\text { familiale lors } \\
\text { du transfert } \\
\end{array}$ & $\begin{array}{l}\text { Statut } \\
\text { juridique }\end{array}$ & $\begin{array}{l}\text { Activité lors } \\
\text { du transfert }\end{array}$ \\
\hline Andrew & $\begin{array}{l}1989 \text { (Londres) } \\
2002 \text { (Ardèche) }\end{array}$ & $\begin{array}{l}48 \text { ans, } \\
\text { célibataire }\end{array}$ & EI & $\begin{array}{l}\text { Création et fabrication } \\
\text { de chapeaux pour } \\
\text { dames }\end{array}$ \\
\hline Dee & $\begin{array}{c}1991 \text { (San Francisco) } \\
1998 \text { (Ardèche) }\end{array}$ & $\begin{array}{l}50 \text { ans, } \\
\text { célibataire }\end{array}$ & EI & $\begin{array}{l}\text { Agence de traduction } \\
\text { multilingue }\end{array}$ \\
\hline $\begin{array}{l}\text { Jean- } \\
\text { François }\end{array}$ & $\begin{array}{l}1988 \text { (Nord) } \\
2003 \text { (Ardèche) }\end{array}$ & $\begin{array}{c}50 \text { ans, marié, } 1 \\
\text { enfant }\end{array}$ & $\begin{array}{l}\text { SARL (puis } \\
\text { EI en 2003) }\end{array}$ & $\begin{array}{l}\text { Conseils en } \\
\text { organisation, } \\
\text { normalisation } \\
\text { et gestion } \\
\text { des risques } \\
\end{array}$ \\
\hline Jean-Louis & $\begin{array}{l}1991 \text { (Paris) } \\
1998 \text { (Gers) }\end{array}$ & 46 ans, marié & SARL & $\begin{array}{l}\text { Réalisation } \\
\text { de CD-Rom et produits } \\
\text { multimédias }\end{array}$ \\
\hline Laurent & $\begin{array}{l}2000 \text { (Belgique) } \\
2003 \text { (Ardèche) }\end{array}$ & $\begin{array}{l}33 \text { ans, marié, } 4 \\
\text { enfants }\end{array}$ & EI & $\begin{array}{l}\text { Conception d'outils } \\
\text { informatiques pour la } \\
\text { formation à distance } \\
\end{array}$ \\
\hline Marc & $\begin{array}{c}2003 \text { (Aix en Provence) } \\
2006 \text { (Ardèche) }\end{array}$ & 33 ans, marié & SARL & $\begin{array}{l}\text { Services et solutions } \\
\text { de gestion de parcs } \\
\text { informatiques et de } \\
\text { help desk }\end{array}$ \\
\hline Marie & $\begin{array}{l}2003 \text { (Annecy) } \\
2004 \text { (Ardèche) }\end{array}$ & $\begin{array}{l}54 \text { ans, } \\
\text { célibataire }\end{array}$ & $\begin{array}{l}\text { EI (puis } \\
\text { SARL en } \\
\text { 2006) }\end{array}$ & $\begin{array}{l}\text { Conseils en gestion } \\
\text { de patrimoine }\end{array}$ \\
\hline Nancy & $\begin{array}{c}1989 \text { (Toronto) } \\
2000 \text { (Gers) }\end{array}$ & 50 ans, mariée & EI & $\begin{array}{l}\text { Conseils en } \\
\text { muséologie }\end{array}$ \\
\hline Nigel & $\begin{array}{l}2001 \text { (Londres) } \\
2004 \text { (Ardèche) }\end{array}$ & 45 ans, marié & EI & $\begin{array}{l}\text { Création de sites } \\
\text { Internet, gestion de } \\
\text { bases de données }\end{array}$ \\
\hline Stéphane & $\begin{array}{c}2000 \text { (Montpellier) } \\
2003 \text { (Vercors) } \\
2004 \text { (Ardèche) }\end{array}$ & $\begin{array}{c}32 \text { ans, marié, } 3 \\
\text { enfants }\end{array}$ & $\begin{array}{l}\text { SARL (puis } \\
\text { EURL en } \\
\text { 2002) }\end{array}$ & $\begin{array}{l}\text { Traduction } \\
\text { français/anglais de } \\
\text { documentations } \\
\text { informatiques }\end{array}$ \\
\hline
\end{tabular}

La sélection des 10 cas analysés a été réalisée de manière à limiter la variabilité des situations étudiées (Yin, 2003). Ces 10 cas forment un sous-groupe homogène des transferts de TPE. Outre les caractéristiques se rattachant à leur trajectoire spatiale commune, les 10 cas retenus partagent en effet une quasi-absence d'employés. Au moment de l'étude, les dirigeants sont seuls dans l'entreprise et, même si certains

${ }^{4}$ Nous avons retenu la date de déménagement comme date de transfert de l'entreprise. Administrativement, ce dernier a généralement eu lieu quelques mois voire quelques années après (jusqu'à 4 ans après pour Dee). Pourtant, il apparaît que c'est bien à partir de son déménagement effectif que l'entrepreneur se trouve confronté aux problématiques de changement de milieu. 
avaient auparavant des salariés et/ou envisagent d'en embaucher dans un futur proche, ils étaient également les seuls concernés par le transfert. En l'absence de tout salarié, la décision de transfert n'est pas le reflet d'un compromis entre des attentes personnelles multiples mais bien l'expression des capacités et de la volonté de l'entrepreneur et de lui seul. Cependant, au sein de ce sous-groupe homogène, les cas sélectionnés sont volontairement très différents. Nous pensons ainsi être en mesure de prendre en compte la variété des problématiques liées à ce type de transferts. En ce qui concerne les caractéristiques sociodémographiques des enquêtés, notre échantillon est composé à la fois de personnes célibataires (3) et en couple (7), avec (3) ou sans (7) enfants à charge. Lors du transfert, le dirigeant était en moyenne âgé de 44 ans avec une variabilité importante (voir tableau 1). L'ampleur géographique du déplacement lors du transfert varie également. Un des enquêtés (Stéphane) a d'ailleurs enchaîné deux transferts (dans le Vercors puis en Ardèche). Le transfert de l'entreprise est plus ou moins récent: entre 1998 et 2006. L'entreprise était alors âgée de 6,6 ans en moyenne mais notre échantillon présente une diversité de situations significative : les entreprises avaient entre 1 et 15 ans lors de leur transfert. Le statut juridique est majoritairement l'entreprise individuelle (EI dans le tableau $1 ; 6$ cas). Seules 4 entreprises étaient des SARL lors du transfert et deux d'entre elles sont par la suite devenues individuelles (EI ou EURL). Il s'agit en effet de deux dirigeants (Jean-François et Stéphane) n'ayant conservé qu'une partie de leur activité initiale à l'issue du transfert. Enfin, les activités des entreprises sont, hormis un artisan d'art, des activités intellectuelles : traduction (2 cas), conseils aux entreprises ( 2 cas) ou aux particuliers ( 1 cas), prestations de services informatiques (4 cas).

Les données ont été recueillies par entretiens semi-directifs rétrospectifs. Ces entretiens ont été enregistrés puis retranscrits. Le guide d'entretien permettait, entre autres, à l'enquêté de présenter son entreprise et d'expliciter les raisons du transfert et les conséquences de ce dernier (notamment sur la thématique des relations avec le milieu d'origine et le milieu d'accueil). Pour l'analyse des données, nous avons procédé à un codage thématique.

\section{Une typologie des logiques de disjonction avec le milieu d'origine}

L'analyse des données empiriques que nous avons recueillies conjuguée à l'analyse de la littérature mobilisée permet de mettre à jour les différents facteurs qui ont été déterminants dans les décisions de transfert étudiées et de mieux comprendre la manière dont ces facteurs s'articulent entre eux. De manière générale, deux grandes catégories de facteurs explicatifs doivent être distinguées. Il s'agit en premier lieu des facteurs permissifs, liés pour l'essentiel à la nature de l'activité des TPE étudiées, qui ont rendu leur transfert faisable techniquement et potentiellement viable économiquement (3.1.).

La décision finale de transfert et la sélection, dans ce cadre, d'un nouveau lieu d'implantation résultent ensuite d'un système complexe d'interactions entre, d'une part, les facteurs permissifs et, d'autre part, la situation et les aspirations personnelles et professionnelles de l'entrepreneur qui vont l'inciter à saisir (ou non) l'opportunité qui s'offre à lui de réaliser la disjonction spatiale avec le milieu local où il a créé son entreprise (3.2.). Une étude attentive des retranscriptions de nos entretiens nous a permis de faire émerger trois grands types de logiques de disjonction au sein de l'échantillon (3.3. à 3.5.). 


\subsection{Les facteurs permissifs de la mobilité lointaine}

L'existence même du phénomène de transfert de TPE d'un milieu urbain vers un milieu rural isolé est le reflet d'un premier ensemble de déterminants liés aux caractéristiques de l'activité des entreprises concernées. En effet, les facteurs mis en avant dans la littérature pour expliquer la forte tendance à l'inertie spatiale des TPE et présentés plus haut peuvent être considérés, en creux, comme des facteurs permissifs facilitant la mobilité géographique des entreprises ou, pour reprendre l'expression employée par Stam (2007), leur « flexibilité spatiale».

Les recherches existantes indiquent ainsi indirectement que le transfert de TPE vers le milieu rural concerne avant tout des activités de services aux entreprises ou aux particuliers nécessitant peu d'investissements dans des actifs immobiliers ou dans des équipements difficiles à déplacer. Ces activités n'étant pas physiquement liées à une localisation, les auteurs anglo-saxons les qualifient, ainsi que les entreprises qui les exercent, de footloose (Stam, 2007).

L'absence de contrainte de proximité géographique avec la clientèle associée au développement des technologies de communication constitue un deuxième élément clé.

\section{Les facteurs permissifs de la mobilité lointaine}

Laurent exerce, au sein de son entreprise, une activité de développement d'outils informatiques pour la formation à distance : C'est une activité de service qui ne me demandait pas - comment dire - une proximité importante vis-à-vis du client. Donc, c'est ça qui m'a permis de m'installer un peu où je voulais.

Cependant, sur ce dernier point, quatre situations doivent être distinguées en fonction de la dispersion de la clientèle et de l'importance des relations de face à face avec les clients.

Tableau 2 : L'absence de contrainte de proximité géographique avec la clientèle : différents cas de figure

\begin{tabular}{|c|c|c|c|}
\cline { 3 - 3 } \multicolumn{2}{c|}{} & \multicolumn{2}{c|}{ Importance des relations de face à face avec la clientèle } \\
\cline { 3 - 4 } \multicolumn{2}{c|}{} & Faible & Forte \\
\hline $\begin{array}{c}\text { Clientèle } \\
\text { avant le } \\
\text { transfert }\end{array}$ & Concentrée & Nigel, Dee & Jean-Louis, Nancy \\
\cline { 2 - 4 } & Dispersée & Laurent, Stéphane & Andrew, Jean-François, Marc, Marie \\
\hline
\end{tabular}

La situation où le départ est le plus aisé est bien sûr celle où la clientèle est à la fois dispersée et nécessite peu d'interactions physiques. À l'opposé, quand, avant le transfert, la clientèle est concentrée dans le milieu d'origine avec des relations de face à face importantes, le transfert de l'entreprise semble plus difficile et implique en tout cas un changement, soit de clientèle (Jean-Louis), soit de mode de gestion des relations avec cette dernière (Nancy). Dans les deux autres cas, une contrainte de proximité géographique 
demeure même si le dirigeant peut avoir l'impression, lors de la prise de la décision de transfert, de pouvoir s'affranchir de cette dernière.

Cependant, ces facteurs permissifs seuls ne permettent pas d'expliquer le transfert vers une zone rurale isolée. Il est également nécessaire de prendre en compte les motivations de l'entrepreneur (Stam, 2007). En effet, les entreprises étudiées étant uniquement constituées de leurs fondateurs, elles sont considérées par ces derniers comme une partie intégrante de leur espace privatif (Torrés, 2003) et la décision de transfert est donc le reflet de leurs préférences individuelles à la fois sur le plan personnel et sur le plan professionnel.

\subsection{Des démarches mêlant étroitement logiques professionnelles et logiques personnelles}

Chacune des décisions de transfert étudiées résulte d'un jeu d'interactions complexe entre des motivations appartenant à la sphère personnelle des décideurs et à leur sphère professionnelle. L'analyse des données empiriques collectées lors des entretiens avec les 10 entrepreneurs de notre échantillon a toutefois permis de repérer des régularités dans les logiques qui ont présidé au choix de la disjonction géographique.

La principale ligne de partage qui est apparue parmi nos cas ne tient pas strictement, comme cela a été souligné dans nombre de travaux antérieurs (voir Reix, 2008, pour un exemple) au poids respectif des déterminants personnels et des déterminants professionnels dans les logiques de disjonction. Conformément à ce qu'avance Torrés (2003), il apparaît en effet que les deux sphères sont très étroitement imbriquées. Le premier axe de différentiation des cas étudiés s'appuie plutôt sur la manière dont l'entrepreneur envisage le transfert au regard de la trajectoire personnelle et professionnelle qu'il a suivi jusque-là.

Cette ligne de partage nous a conduits à distinguer dans notre échantillon :

- d'un côté, des situations où les entrepreneurs apparaissaient pleinement satisfaits de leur situation personnelle et de leur accomplissement professionnel avant le transfert. Ce dernier s'inscrit alors dans le prolongement de cette réussite initiale perçue. L'entrepreneur souhaite l'approfondir en associant à sa démarche entrepreneuriale une amélioration de ses conditions de vie et de celles de ses proches (3.5.)

- de l'autre côté, des situations dans lesquelles les entrepreneurs ont été conduits à envisager un déménagement et un transfert de leur affaire en zone rurale en raison d'une insatisfaction croissante face à leur situation professionnelle et personnelle. Dans ce deuxième type de situation, le transfert est d'abord envisagé par l'entrepreneur comme un moyen d'infléchir sa trajectoire et celle de son entreprise, en prenant du recul (aux sens physique et relationnel du terme) par rapport à son milieu d'origine. Au sein de cette deuxième catégorie, deux types de logiques doivent être distinguées selon la manière dont l'entrepreneur ressent sa situation dans son milieu de départ au moment où il prend la décision du transfert. Dans le premier type, le transfert a pour origine l'échec relatif de la démarche entrepreneuriale auquel se conjuguent parfois des déboires personnels importants (3.3.). La disjonction géographique est alors vécue comme une rupture offrant la possibilité de repartir sur de nouvelles bases, en particulier sur le plan professionnel, dans un environnement perçu comme globalement moins oppressant. Dans le deuxième type, l'entrepreneur se trouve plutôt dans une situation de réussite professionnelle dans son milieu urbain d'origine, mais cette situation est jugée quelque peu envahissante pour sa vie 
personnelle et familiale. Le transfert est dans ce cas envisagé comme un moyen de rééquilibrer vie privée et vie professionnelle en prenant volontairement du recul par rapport aux réseaux spécifiquement liés à cette dernière (3.4.)

Le tableau 3 permet de visualiser l'appartenance de chacun des 10 cas étudiés aux trois grands types de logique de disjonction spatiale qui viennent d'être distingués. Ces logiques sont présentées de manière plus détaillée dans les trois sections suivantes.

Tableau 3 : Les principaux types de logiques de disjonction spatiale de l'échantillon

\begin{tabular}{|c|c|c|c|}
\cline { 3 - 4 } \multicolumn{2}{c|}{} & \multicolumn{2}{c|}{$\begin{array}{c}\text { Effet recherché du transfert sur la trajectoire } \\
\text { de l'entrepreneur }\end{array}$} \\
\cline { 3 - 4 } \multicolumn{2}{c|}{} & Inflexion & Continuité \\
\hline \multirow{2}{*}{$\begin{array}{c}\text { Ressenti de } \\
\text { la situation } \\
\text { professionnelle } \\
\text { et personnelle } \\
\text { avant le } \\
\text { transfert }\end{array}$} & Réussite & $\begin{array}{c}\text { Type 2: Mettre de la } \\
\text { distance } \\
\text { Nancy, Andrew, Nigel, } \\
\text { Laurent }\end{array}$ & $\begin{array}{c}\text { Type 3: Prolonger } \\
\text { la démarche entrepreneuriale } \\
\text { Jean-Louis, Dee, Marc }\end{array}$ \\
\cline { 2 - 4 } & $\begin{array}{c}\text { Echec } \\
\text { relatif }\end{array}$ & $\begin{array}{c}\text { Type 1 : Prendre } \\
\text { un nouveau départ } \\
\text { Jean-François, Stéphane, } \\
\text { Marie }\end{array}$ & \\
\hline
\end{tabular}

\subsection{Type 1 : Prendre un nouveau départ}

Le premier type de logique correspond à un comportement de fuite devant une situation première qui est appréhendée comme un échec relatif. Sur le plan professionnel, l'entrepreneur ne se sent pas ou plus en mesure d'atteindre les objectifs qu'il s'est initialement fixés.

La situation d'échec ressentie par l'acteur ne vient donc pas nécessairement d'un échec

\section{Une situation d'échec relatif}

Stéphane explique ainsi pourquoi il a décidé de quitter la société qu'il avait fondée à Montpellier avec 3 autres personnes pour s'installer à son compte dans le Vercors puis en Ardèche: D'abord, je me suis séparé, je vivais avec une des personnes qui était aussi actionnaire de l'entreprise et donc on s'est séparé. Mais, ce qui s'est passé surtout, et donc qui a été l'élément déclencheur pour moi, ça a été le fait que mon comptable ait piqué dans la caisse de l'entreprise et qu'il ne fasse même pas les tâches administratives ou de gestion telles que je les lui demandais... Et donc, comme je n'arrivais pas à lui faire entendre raison, on a fini sur un conflit assez important et j'ai dit « ben si c'est comme ça. »Comme c'était moi qui étais à l'origine du contrat, ben j'ai dit « si c'est comme ça, puisque je n'arrive pas à avoir quelque chose qui correspond à ce que je souhaite, c'est-à-dire une entreprise qui fait pas de blagues, pas de fausses déclarations, pas de machin etc, et bien je me barre. Et je prends mon contrat et je m'en vais avec. » 
commercial ou financier qui mettrait en danger la survie à court terme de son entreprise, mais plutôt de la sensation que la situation lui échappe et qu'il ne contrôle plus ses réseaux personnels et d'affaires qui ont été jusque-là si utiles à la réussite de son projet. Il se retrouve alors confronté à des demandes de réciprocité économiques ou non-économiques qui finissent par nuire à l'activité de son entreprise, à sa satisfaction au travail et à son équilibre vie professionnelle - vie privée (Granovetter, 1995; Uzzi, 1997; Adler et Kwon, 2002; Huault, 2004). Il en vient à penser que prendre du recul physiquement par rapport à ce milieu professionnel qui devient trop pesant serait la meilleure solution pour remédier aux difficultés rencontrées à la tête de son affaire. Cette volonté de prendre du recul se traduit également, dans deux des trois cas correspondant à ce type de démarche au sein de notre échantillon, par la dissolution de la société créée dans le milieu urbain d'origine et la création d'une nouvelle structure dans le milieu rural d'arrivée. Ces recréations s'accompagnent toutefois de la conservation d'une partie de la clientèle et de l'activité des sociétés antérieures.

\section{Un milieu d'origine devenu hostile}

Jean-François explique que son souhait de se débarrasser des deux sociétés qu'il possédait dans le Nord pour recréer ensuite une nouvelle entreprise en Ardèche a été en partie motivé par les difficultés de paiement qu'il a rencontré avec l'un de ses plus gros clients : Donc, j'ai dit, bon, pourquoi vouloir embaucher et faire des employés pour justement plein de risques. Je suis en train de gérer les risques des autres et j'étais en train de m'en faire tout plein.

Bien que des considérations plus générales de qualité de vie soient mises en avant par les entrepreneurs du type 1, le milieu rural isolé est avant tout présenté, dans leur discours, comme un refuge, un havre de paix où il leur est toujours possible de se retirer pour travailler « dans le calme » (Jean-François) et « tranquillement » (Marie).

\section{Un nouvel ordre de priorité qui valorise les souhaits de la conjointe}

Pour faire mon boulot et tout ça, il faut être dans le calme. Mais que je sois n'importe où en France, de toute façon, j'avais une gêne, c'est que mes clients sont dans toute la France. Donc moralité, que je parte du Nord, que je parte d'Ardèche pour faire mes tournées en France, peu m'importe. [...] En plus, j'allais dire, la deuxième chose, c'est qu'on pensait y être dans 5-6 ans parce que ma femme avait demandé sa mutation et on lui avait dit « ben, fonctionnaire, il faut 5-6 ans ». Et 15 jours après, il y avait une place à 7-8 km. Donc, c'était soit le choix de prendre tout de suite, soit de reculer d'autant. (Jean-François)

Les transferts regroupés au sein de cette classe sont d'abord pensés en fonction des "facteurs d'expulsion" (Van Dijk et al., 2000) qui conduisent le dirigeant à fuir son milieu d'origine et une vision quelque peu idéalisée de la qualité de vie à la campagne (Chevalier, 2003). La confrontation à la réalité de l'implantation en zone rurale isolée 
nécessite de ce fait des ajustements a posteriori dans la conduite de l'affaire, voire des modifications dans la nature des activités de l'entreprise. Ces ajustements ne remettent toutefois pas fondamentalement en question le projet initial.

\section{Le caractère de nouvel arrivant comme frein à la constitution d'un réseau social et professionnel dans le milieu d'accueil}

Mon premier handicap, c'est que je ne suis pas d'ici, voilà. C'est le premier handicap quand vous vous installez quelque part. On n'est pas d'ici. [...] Bon, il y a beaucoup de clans. Donc, ça, en fait, le fait de ne pas être nés ici, de ne pas avoir vécu ici, on est des pièces rapportées. Donc c'est très difficile de travailler avec des gens qui ont déjà cet a priori. (Marie)

\section{Modifier l'activité pour alléger la contrainte de proximité géographique temporaire}

Après avoir expérimenté une activité de conseils à distance, Jean-François a ajouté à son activité de conseils en organisation celle de vente d'un logiciel, l'objectif étant de limiter les déplacements auprès des clients. J'ai créé des outils avec un partenaire informaticien et au lieu de me déplacer comme je le faisais avant, et bien, ici, je me suis aussi mis en organisme de formation et plutôt que de faire plein de journées de conseil, je vends des logiciels avec une formation qui permettent d'alléger le conseil mais, en même temps, qui donne une aide et les personnes peuvent faire sans moi après.

\subsection{Type 2 : Mettre de la distance avec son milieu d'origine}

Le second type de logiques regroupe des entrepreneurs qui ont vu dans le transfert de leur affaire en zone rurale isolée l'occasion de mettre en œuvre une approche de leur activité professionnelle et, de manière plus générale, une philosophie de vie beaucoup plus hédoniste. Cet hédonisme est d'abord sensible dans leur manière de diriger leur TPE. Dans cette perspective, le transfert et la distance géographique qu'il

\section{Le transfert en milieu rural isolé, une opportunité de mettre en œuvre une vision hédoniste de son métier}

Le cas de Nancy, une consultante en muséologie canadienne qui s'est installée dans le Gers en 2000, est particulièrement représentatif de ce type de logique : Je ne fais pas de marketing, je ne fais pas de publicité. Je suis assez bien connue, alors, même en France, ou même en Allemagne, à cause de mes activités avec ces associations. Mon nom est assez bien connu [...] Je suis venue ici pour m'éloigner aussi, avoir un peu plus de temps pour les projets qui me passionnent, plutôt que d'être dans une grande ville, avec tout ce que ça comprend. 
instaure avec leur milieu d'origine sont perçus par le dirigeant comme des moyens de faire le tri dans son portefeuille de clients et plus largement dans son portefeuille de relations pour ne conserver que celles dans lesquelles il (ou elle) souhaite s'investir réellement :

- soit parce qu'il s'agit de liens forts basés sur des affinités multiples (professionnelles, amicales...). Dans ce cas, la distance géographique peut être vue comme un révélateur permettant de mettre à jour les relations les plus fortes qui sauront résister à l'éloignement géographique ;

- soit parce qu'elles représentent, d'un point de vue professionnel, un intérêt intrinsèque particulièrement prononcé.

Cette démarche rapproche ainsi les entrepreneurs de ce type de la catégorie des « artistes » qui vivent leur métier comme une passion (Jaouen, 2008).

Cette démarche hédoniste englobe l'équilibre entre vie professionnelle et vie privée. Les entrepreneurs du type 2 appréhendent également leur transfert à la campagne comme une opportunité de réorganiser leurs priorités en relativisant l'importance de leur activité professionnelle au profit de leur vie personnelle ou familiale (mise en avant des souhaits du (de la) conjoint(e), du bien-être des enfants et/ou réalisation d'un idéal de vie à la campagne dans le prolongement des premiers tenants de la contre-urbanisation des années 70).

\section{Une volonté d'accorder plus de place aux aspirations personnelles et familiales}

Nigel, qui a transféré son activité de conception de sites Internet et de gestion de base de données de Londres à la campagne ardéchoise, considère que ses préoccupations personnelles et familiales ont joué un grand rôle dans sa décision : Donc, en effet, quelque part, en venant ici, j'ai pensé que mon entreprise serait de moins en moins importante. Et je vois maintenant qu'en effet, ce n'est pas le cas, que mon entreprise elle va être encore importante. Donc, c'est aussi une des raisons pour laquelle je n'ai pas fait beaucoup de recherches avant de venir.

Comme l'indique cette dernière citation, cette catégorie de logiques de transfert est susceptible de s'accompagner d'un manque de préparation particulièrement prononcé, l'entrepreneur pouvant parfois faire preuve d'un excès de confiance dans ses capacités professionnelles pour surmonter les handicaps liés au sous-équipement de la localisation choisie.

\section{Des difficultés postérieures à l'installation mal anticipées par l'entrepreneur}

Les problèmes qu'on a rencontrés, et je suis très contente qu'on les ait rencontrés après, parce que je vous dis que, franchement, je ne sais pas si on aurait pu venir dans le Gers en sachant ces complications. [...] C'est le problème avec le téléphone portable, la couverture est très mauvaise, très mauvaise. En plus, on n'a pas le haut 
débit. On n'aura pas le haut débit d'ici un certain nombre d'années. Alors, je suis toujours en train de... ce matin une heure avec Wanadoo. Pour certains problèmes, c'est très difficile. Ce n'est vraiment pas un endroit où on peut mener une véritable vie professionnelle. Il faut s'adapter avec beaucoup de patience et... Mais il y a aussi un certain manque de services. Il n'y a qu'une très petite entreprise de service de photocopieuse, pour les rapports et les choses comme ça. (Nancy)

\subsection{Type 3 : Prolonger la démarche entrepreneuriale}

La troisième grande logique de disjonction s'appuie non pas sur un quelconque échec ressenti dans le milieu d'origine (comme le type 1) mais plutôt sur le succès initial au regard des objectifs entrepreneuriaux d'origine. Cependant, ce succès ne conduit pas le dirigeant à mettre entre parenthèses son entreprise au profit d'un projet de vie comme dans le type 2. Au contraire, il l'incite à approfondir sa démarche entrepreneuriale qui trouve souvent sa source dans une insatisfaction avec son poste précédent (Stam, 2007). Le changement total de cadre de vie exprime alors la volonté de rompre avec un environnement urbain sur lequel l'entrepreneur porte un regard globalement négatif (entassement urbain, contraintes temporelles fortes et conflictuelles, problèmes de transports, notamment dans les déplacements domicile-travail, etc.) auquel il oppose une vision quelque peu enjolivée de l'espace rural isolé.

\section{Le rejet de la ville}

Après 15 ans aux États-Unis, ça me suffisait. Trop polluée, trop d'insécurité, trop de... c'est sale, c'est trop... tout va trop vite, on n'a pas le temps. (Dee)

J'en avais marre de perdre du temps dans les embouteillages... À Paris, on est coincé tout le temps dans les embouteillages, j'en avais ras-le-bol de prendre la voiture. J'avais... j'avais envie de partir. [...] Ça me pesait d'habiter, même si notre appartement était sympa, j'avais quand même l'impression, à la fin, d'habiter dans une cage à lapin. (Jean-Louis)

Il serait dès lors possible d'assimiler ce type de raisonnement au courant du «lifestyle entrepreneur » (Johannisson, 2004). Toutefois, ce type d'entrepreneur, qui est surtout apparu dans le secteur du tourisme (gîtes ruraux ou chambres d'hôtes, par exemple) correspond à des individus pour lesquels la recherche de la qualité de vie prime sur la rentabilité économique. Or, tel n'est pas le cas ici. La démarche de ces entrepreneurs s'appuie plutôt sur leur réussite antérieure qui les incite à penser que, s'ils sont en mesure de bien gérer leur affaire en milieu urbain, ils pourront tout aussi bien, grâce aux facteurs permissifs évoqués plus haut et à l'aide de connexions à haut-débit à Internet, gérer la relation à distance avec leurs clients ou leurs partenaires en affaires tout en profitant d'un cadre de vie jugé plus agréable que celui de leur milieu d'origine. Le transfert n'est pas 
perçu comme entravant la démarche entrepreneuriale. Au contraire, le développement de l'entreprise, voire la croissance en taille, fait partie des effets recherchés du transfert au même titre que l'amélioration de la qualité de vie.

\section{Un transfert qui ne freine pas le développement de l'entreprise}

Marc est positionné sur une activité de niche avec un marché national. Le transfert de son entreprise en Ardèche n'est pas vu comme un frein à son développement. L'objectif c'était aussi m'étendre, grossir. [...] je suis déjà intervenu dans la région, je sais qu'il y a un potentiel d'activité qui existe. Les impacts du transfert sont d'ailleurs relativement faibles : c'est le cas de clients à [Ville d'origine], depuis qu'ils savent que j'ai déménagé, ils tolèrent beaucoup plus que je travaille à distance. Il a conservé son équipe de conseillers (comptable, banquier) dans son milieu d'origine et échange avec eux par téléphone. Cependant, il souligne que pour les nouveaux clients, les déplacements permettant une proximité géographique temporaire demeurent nécessaires. Il est d'ailleurs les deux tiers de son temps en déplacement.

Dans ce cas, il est possible d'envisager que l'idée du transfert en milieu rural ait été planifiée de longue date, voire même qu'elle soit présente dans l'esprit de l'entrepreneur dès la création de l'entreprise. La réflexion préalable au transfert et sa préparation sont alors effectuées de manière plus approfondie et mûrie, ce qui n'empêche pas que d'éventuelles mauvaises surprises puissent surgir de façon inopinée, une fois le transfert effectué.

\section{Une décision mûrie et prudente}

Jean-Louis signale qu'une possibilité de réversibilité de la décision avait été envisagée même si cette alternative, qui aurait été considérée par l'entrepreneur comme un échec, avait au final peu de chance d'être choisie, quoiqu'il arrive :... Sur le plan juridique, sur le plan statutaire de la société, j'ai ouvert, comme j'avais gardé une adresse postale à Paris, j'ai ouvert, ici, un établissement secondaire. Pendant longtemps, les premières années, comme je ne savais pas si ça allait marcher ou pas, si on n'allait pas être obligés de revenir à un moment ou à un autre [...] Mais, bon, moi, dans ma tête, c'était déjà joué, j'avais pas l'intention de remonter sur Paris.

\section{Implications et prolongements}

Notre étude empirique apporte un éclairage sur un phénomène encore peu étudié : les transferts de TPE d'un milieu urbain vers un milieu rural isolé. Elle est en ce sens riche d'implications potentiellement utiles d'un point de vue académique, dans la perspective d'une amélioration de la compréhension des déterminants, mais aussi des conséquences de ces transferts à la fois sur les TPE et sur les territoires d'accueil choisis (4.1. et 4.2.). Elle est également porteuse d'enseignements intéressants pour les acteurs locaux (collec- 
tivités locales, chambres de commerce et d'industrie, chambres des métiers et de l'artisanat...) désireux d'améliorer les structures d'accueil et les dispositifs d'accompagnement destinés aux entrepreneurs dans les zones rurales isolées (4.3.).

\subsection{Le rôle de l'attitude de l'entrepreneur par rapport à ses réseaux dans la décision de transfert}

Les résultats de notre étude empirique montrent en premier lieu que, au-delà de l'étroite imbrication des logiques professionnelles et personnelles dans la décision de transférer une TPE et dans le choix d'une destination particulière pour ce faire, l'attitude de l'entrepreneur vis-à-vis de ses réseaux de relations interpersonnelles, notamment des réseaux qu'il mobilise pour créer et faire vivre son affaire, ainsi que les modifications de cette attitude au fil du temps jouent un rôle déterminant dans l'explication des décisions de transfert d'un milieu urbain vers une zone rurale isolée. Cette attitude peut être appréhendée comme un arbitrage entre logiques d'encastrement et de désencastrement. Au vu de nos résultats, cet arbitrage, loin d'être stable dans le temps, peut subir des évolutions importantes.

La littérature indique que l'entrepreneur qui souhaite réussir doit parfois s'affranchir, se désencastrer, au moins partiellement, de son environnement social d'origine dont les attentes et les valeurs ne sont pas nécessairement en phase avec la logique économique de création d'entreprise (Granovetter, 1995 ; François-Noyer et Philippart, 2005).

Notre travail complète ces enseignements en montrant que, dans le cas particulier des transferts de TPE d'un milieu urbain vers un milieu rural isolé, certains entrepreneurs décident, de leur propre chef, de prendre de la distance non seulement avec leurs réseaux sociaux, mais également avec leurs réseaux professionnels, ces deux types de réseaux se recouvrant plus ou moins largement au sein de relations multiplexes dans les cas étudiés. Cette prise de distance se traduit d'abord et avant tout physiquement, au travers du transfert dans l'espace géographique de la TPE conjugué au déménagement de l'entrepreneur lui-même. Cette mobilité géographique durable est également appréhendée par les entrepreneurs eux-mêmes, notamment au sein du type 1 de notre typologie (dans le tableau 3, « Prendre un nouveau départ ») et, dans une moindre mesure, du type 2 (dans le tableau 3, «Mettre de la distance »), comme une forme de désencastrement par rapport aux réseaux construits au fur et à mesure de leur parcours antérieur en milieu urbain. Dans cette perspective, l'isolement géographique des zones rurales choisies, loin d'être un repoussoir, est ainsi valorisé par les entrepreneurs interrogés.

Cette volonté de désencastrement doit toutefois être relativisée car même les entrepreneurs ayant suivi une logique de type 1 souhaitent conserver une partie des liens forts tissés avant leur transfert. Les modifications attendues suite aux transferts s'apparentent plutôt, dans la plupart des cas étudiés, à une évolution vers une situation de "mixed embeddedness » (Kloosterman et al., 1999) comparable à la situation des entrepreneurs immigrants pour lesquels il est important d'être insérés non seulement dans des réseaux ethniques (donc en lien avec leurs origines), mais également dans des réseaux professionnels au sein de leur milieu d'accueil. En effet, le sur-encastrement dans un réseau ethnique peut conduire à des effets négatifs tels que des pressions pour fournir une aide financière à la communauté ou embaucher des personnes issues de cette communauté 
(Razin, 2002). Dans les cas étudiés, le transfert constitue une manière d'opérer un tri, dont la sélectivité n'est toutefois que partiellement contrôlée par les entrepreneurs, parmi les relations interpersonnelles constituées préalablement. Il est aussi appréhendé comme une opportunité de se reconstituer de nouveaux réseaux dans le milieu d'accueil rural. Ainsi, nombre de nos répondants envisageaient, dès le départ, une réorientation de leur activité vers une clientèle plus locale ou le démarrage d'une activité annexe, telle que la gestion d'un gite ou de chambres d'hôtes, leur permettant d'améliorer leur ancrage local.

Il convient toutefois de noter que les cas étudiés présentent une différence majeure avec la situation des entrepreneurs immigrés : les réseaux développés dans le milieu d'origine ne semblent pas intrinsèquement incompatibles avec le développement de l'entreprise. Certains des entrepreneurs interrogés avaient d'ailleurs constitué des réseaux inter-organisationnels forts et denses avant le transfert. Leur intention initiale est de conserver au moins une partie de ces réseaux inter-organisationnels. Le transfert est plutôt destiné, notamment dans les logiques de type 1, à se délester d'une partie de ces réseaux et, dans tous les cas, alléger, au travers de la distance géographique qui est instaurée avec eux, la pression et les contraintes qui leur sont associées de manière à pouvoir mieux conjuguer vie professionnelle et vie personnelle et familiale, mais également en vue de libérer suffisamment de temps et de moyens pour se constituer des réseaux dans le milieu rural d'implantation afin de mettre pleinement en œuvre le projet de vie individuel sous-jacent au transfert.

Les cas d'entrepreneurs étudiés militent également pour une remise en cause plus radicale des modèles de coévolution des TPE et des réseaux de leur dirigeant tels que celui de Butler et Hansen (1991). Ces modèles apparaissent en effet, au regard du contenu de nos entretiens, à la fois trop linéaires et trop monolithiques. Trop linéaires car ils ne prennent pas en compte la possibilité de survenance, dans les parcours entrepreneuriaux, d'événements perturbateurs de l'évolution des réseaux tels qu'un transfert d'un milieu urbain vers une zone rurale isolée, ces événements perturbateurs ne relevant pas de chocs exogènes indépendants de la volonté de l'entrepreneur mais au contraire de décisions délibérées de sa part. Trop monolithiques car les parcours des entrepreneurs interrogés indiquent que ces derniers se retrouvent insérés, suite à leur transfert, dans deux réseaux (un réseau préexistant au transfert d'une part et un réseau constitué postérieurement dans le milieu rural d'accueil d'autre part) fortement disjoints l'un par rapport à l'autre dont l'inscription spatiale, les finalités et les potentialités de mobilisation à des fins professionnelles différent sensiblement. Notre travail de recherche pose ainsi clairement la question des conditions de coexistence et de coévolution de ces deux types de réseaux, ces conditions étant susceptibles de varier selon la nature des logiques ayant présidé aux transferts des TPE concernées.

\subsection{La mise à jour de la diversité et du caractère évolutif des logiques de disjonction géographique observées}

Le deuxième grand enseignement de notre étude réside dans la mise à jour d'une diversité des logiques de disjonction géographique adoptées par les entrepreneurs qui ne suit pas nécessairement les découpages mis en évidence par les travaux préexistants. Ainsi, les transferts étudiés découlent de situations de départ différentes en termes de ressenti personnel 
et professionnel vis-à-vis des milieux d'origine, mais également en termes de perceptions du milieu rural isolé choisi pour s'implanter. Dans ces conditions, les attentes des entrepreneurs par rapport à leur nouveau milieu d'accueil ne seront pas les mêmes selon leur position dans notre typologie des logiques de disjonction (voir tableau 3, section 3.2.). Les attentes des entrepreneurs relevant des types 2 et 3 (première ligne du tableau 3, « Mettre de la distance » et « Prolonger la démarche entrepreneuriale ») auront tendance à concerner en priorité la qualité de vie et la sphère personnelle, alors que les entrepreneurs relevant du premier type (deuxième ligne du tableau 3, « Prendre un nouveau départ ») seront plus prompts à considérer leur milieu d'accueil comme une source d'opportunités professionnelles et commerciales nouvelles. Notre typologie rejoint ainsi la distinction établie par Bossuet (2011) entre, d'une part, ceux qu'il qualifie de « nouveaux ruraux » qui souhaitent s'installer à la campagne pour des raisons de qualité de vie tout en conservant des relations économiques étroites avec leur milieu urbain d'origine (les types 2 et 3 de notre typologie) et, d'autre part, des « néo-ruraux » qui recherchent une rupture socio-économique plus franche avec l'urbanité (le type 1 de notre typologie).

Nos résultats laissent également transparaître une certaine naïveté, voire même, dans certains cas, une franche impréparation des entrepreneurs interrogés par rapport aux zones rurales où ils s'installent.

Or, le transfert d'une TPE d'un milieu urbain vers un milieu rural est rarement indolore pour l'entreprise et en particulier pour le réseau d'affaires dans lequel elle est insérée. Une analogie peut ici être faite avec le cas des migrations individuelles. En effet, le déménagement, et la rupture géographique qu'il entraîne, est un événement particulièrement perturbateur du réseau social d'un individu (Grossetti, 2001). Dans une étude sur les jeunes migrants des régions rurales vers les villes au Québec, Assogba et al. (2000) montrent que la migration s'accompagne d'un processus de reconstitution du réseau social. Ce processus se caractérise, au début, par une tentative de maintien des contacts avec le milieu d'origine (qui se termine généralement par la rupture de nombreux liens amicaux et le maintien des seules relations familiales), puis la reconstruction d'un nouveau réseau social dans le milieu d'accueil.

Perçue comme largement surmontable par l'utilisation des Technologies de l'Information et de la Communication (et l'ubiquité qu'elles permettent) et/ou par le recours à une proximité géographique temporaire (Torre, 2009), la contrainte de proximité géographique (notamment avec les clients) nécessaire au maintien du réseau initial apparaît parfois sous-évaluée. En effet, l'éloignement géographique (induit par le transfert) entre l'entrepreneur et son réseau d'affaires s'accompagne bien souvent d'un éloignement cognitif, les deux acteurs ne partageant plus vraiment le même cadre de référence. Cet éloignement est particulièrement problématique pour les entrepreneurs inscrits dans une démarche de «nouveaux ruraux » (Bossuet, 2011). Ces nouveaux ruraux, dont la migration a été guidée en grande partie par la recherche d'une qualité de vie meilleure et d'un rythme de vie moins stressant, peuvent ne plus percevoir avec la même urgence et la même importance les demandes pressantes de leurs relations d'affaires restées dans un environnement urbain.

Ce décalage peut conduire, voire parfois contraindre l'entrepreneur à réorienter, au moins partiellement, son activité professionnelle de manière à s'adresser plus directement aux attentes d'une clientèle locale. Des tentatives de réorientation de ce type ont été relevées, lors de nos entretiens, dans la majorité des cas étudiés. 


\subsection{Propositions pour des dispositifs d'accompagnement adaptés}

Les logiques bien particulières qui président aux transferts de TPE de milieux urbains vers des zones rurales isolées requièrent la mise en place de dispositifs d'accompagnement adaptés. Ces dispositifs sont d'autant plus importants pour les aires géographiques concernées que la littérature a souligné la place déterminante que les entrepreneurs extérieurs sont susceptibles de jouer dans leur revitalisation économique (Chevalier, 2000).

Nos résultats incitent en premier lieu à concevoir une approche associant l'accompagnement professionnel (afin, notamment, de favoriser l'intégration de la TPE au tissu économique local) et l'accompagnement individuel de l'entrepreneur et de sa famille pour aider à leur intégration personnelle au sein des milieux ruraux d'accueil.

Par ailleurs, il ne saurait y avoir un seul type de dispositif d'accompagnement qui puisse être considéré comme supérieur en vue de faciliter la réussite de ces entrepreneurs en zone rurale et de maximiser les effets d'entraînement que leur installation peut avoir sur le tissu économique local. Les résultats obtenus invitent au contraire à adapter les dispositifs d'accompagnement aux principales caractéristiques des logiques de transfert suivies par les entrepreneurs et, donc, aux attentes qu'ont ces derniers vis-à-vis de leur territoire d'accueil.

Enfin, les collectivités locales ont tout intérêt à favoriser l'intégration professionnelle partielle de ces entrepreneurs nouvellement arrivés dans la mesure où cela peut être un facteur de redynamisation de l'économie locale à la fois directement et indirectement au travers des passerelles que l'entrepreneur peut mettre en place entre ces activités professionnelles locales et les activités préexistantes réalisées en relation avec les réseaux dont il dispose encore dans son milieu d'origine. Ainsi, comme l'observent Kalandaris et Bika (2006), «les entrepreneurs néo-ruraux favorisent l'intégration des communes autrefois reculées dans les réseaux nationaux et globaux de production et de distribution » ( p. 126).

La possibilité, pour les entrepreneurs nouvellement installés, de pouvoir combiner activité locale et activité à destination d'une clientèle extérieure est également de nature, par les effets compensatoires qui peuvent exister entre les deux (notamment en termes de revenus), à favoriser l'ancrage de ces derniers.

\section{Conclusion}

L'analyse de la littérature et l'exploitation des 10 cas d'entrepreneurs ayant transféré leur entreprise d'une agglomération urbaine vers une zone de l'espace rural isolé a permis de montrer que l'étude de ce type de trajectoire spatiale atypique constitue un champ de recherche particulièrement riche et prometteur en raison du caractère révélateur de ces opérations de transfert. Cette recherche contribue ainsi à apporter une meilleure compréhension des déterminants de l'adoption, par certains entrepreneurs, de ce type particulier de parcours géographique et à s'interroger sur les conséquences qui peuvent en résulter pour la santé et le fonctionnement de l'affaire qu'ils dirigent ainsi que pour les territoires d'accueil choisis. Elle a notamment permis de mettre en avant le rôle central joué par les évolutions de l'attitude de l'entrepreneur face à ses réseaux dans la prise de la décision de transfert. Les résultats obtenus indiquent également la diversité des raisonnements ayant conduit à la mise en œuvre de telles opérations et le caractère évolutif des activités et des 
comportements de réseautage des entrepreneurs concernés. Les trajectoires personnelles et organisationnelles étudiées apparaissent particulièrement atypiques au regard des travaux existant sur l'évolution des réseaux de l'entrepreneur. Ces différences peuvent s'expliquer par le fait que notre attention s'est portée sur des entreprises dont la taille demeure très petite (la plupart était, à la date de l'étude, des structures unipersonnelles). Dans ces conditions, les modèles de coévolution des entreprises et des réseaux de l'entrepreneur doivent être amendés par une meilleure intégration de la gestion fortement personnalisée (Torrés, 2003) des TPE et, en conséquence, des objectifs personnels et familiaux de leurs dirigeants. Toutefois, dans un contexte où le potentiel de mobilité de ces TPE et de leurs dirigeants se trouve singulièrement accru par l'apparition et la diffusion de nouvelles technologies mobiles (Urry, 2005), la «conception égocentrée de l'espace » (Torrés, 2003, p. 122) caractéristique des dirigeants de TPE n'est plus systématiquement synonyme d'inertie spatiale, militant ainsi pour la construction de modèles d'évolution des réseaux de l'entrepreneur à la spatialisation moins enracinée dans un espace local déterminé, y compris lorsqu'il s'agit d'un espace urbain ouvert à un large éventail de connexions distantes.

Ces résultats incitent enfin à l'adoption, par les collectivités locales des territoires ruraux désireux d'attirer de nouvelles activités et acteurs économiques, de dispositifs d'accueil et d'accompagnement adaptés.

La présente étude empirique mérite d'être complétée par des travaux centrés sur le même objet de recherche mais réalisés sur des terrains différents, en particulier en termes de zones géographiques étudiées. Ces travaux permettraient de renforcer la compréhension des logiques et des effets des transferts de TPE d'un milieu urbain vers un milieu rural et de mettre à jour de manière plus détaillée les caractéristiques des dispositifs d'accompagnement les plus adaptés à ce type de parcours entrepreneurial. Par ailleurs, des analyses longitudinales seraient riches d'enseignements sur la façon dont évoluent les logiques d'action des dirigeants au fur et à mesure de la mise en œuvre de leur projet de transfert. 


\section{Références}

Anderson A., 2000. Paradox in the Periphery: an entrepreneurial Reconstruction? Entrepreneurship and Regional Development 12 (2), 91-110.

Adler P., Kwon S.W., 2002. Social Capital: Prospects for a new Concept. Academy of Management Review 27 (1), 17-40.

Anderson A. and Jack S., 2002. The Articulation of Social Capital in Entrepreneurial Networks: a Glue or a Lubricant?, Entrepreneurship and Regional Development 11, 198-210.

Assogba Y, Fréchette L., Desmarais D., 2000. Le mouvement migratoire des jeunes au Québec. La reconfiguration du réseau social, un repère pour étudier le processus d'intégration. Nouvelles Pratiques Sociales 13 (2), 65 78.

Bossuet L., 2011.Recomposition sociodémographique des campagnes du sud-ouest de la France : Implications locales et mobilités. Revue Canadienne des Sciences Régionales 34 (4), 135-150.

Brouwer A.E., Mariotti I., Van Ommeren J.N., 2004. The Firm Relocation Decision: an Empirical Investigation. Annals of Regional Sciences 38, 335-347.

Butler J.E., Hansen E.L., 1991. Network Evolution, Entrepreneurial Success, and Regional Development. Entrepreneurship and Regional Development 3 (1), 1-16.

Chanteau J.P., 2001. L'entreprise nomade, localisation et mobilité des activités productives, L'Harmattan, Paris.

Chevalier P., 2000. Dynamiques tertiaires de l'espace rural, Thèse de doctorat de géographie. Université Paul Valery, Montpellier 3.

Chevalier P., 2003. Qualité de vie et logique économique dans les espaces de faible densité. Revue d'Économie Méridionale 51 (201-202), 149-153.

Champion A., 1989, Counterurbanization: the Changing Pace and Nature of Population Deconcentration. Edward Arnold, London.

Elfring T., Hulsink W., 2007. Networking by Entrepreneurs: Patterns of Tie-Formation in Emerging Organizations, Organization Studies 28 (12), 1849-1872.

Figuereido O., Guimaraes P., Woodward D., 2002. Home-field Advantage: Location Decisions of Portuguese Entrepreneurs. Journal of Urban Economics 52, 341-361.

Font E., 2000. Les activités non agricoles dans la recomposition de l'espace rural. L'Harmattan, Paris.

François-Noyer V., Philippart P. 2005. La création d'entreprise par un chercheur fonctionnaire : la gestion du désencastrement. Finance Contrôle Stratégie 8 (1), 49-71.

Granovetter M., 1985. Economic Action and Social Structure: The Problem of Embeddedness, American Journal of Sociology 91 (3), 481-510.

Granovetter M., 1995. The Economic Sociology of Firms and Entrepreneurs. In Portes A. (Ed.), The Economic Sociology of Immigration, Russell Sage Foundation, New York City, 128-165.

Grossetti M., 2001. Les effets de proximité spatiale dans les relations entre organisation: une question d'encastrements. Espaces et Sociétés (101-102), 203-219.

Grossetti M., 2004. Proximities and Embedding Effects. European Planning Studies 16 (5), 613-616.

Hilal M., Schmitt B., 1997. Les espaces ruraux. Une nouvelle définition d'après les relations villes-campagnes. INRA Sciences Sociales, Recherches en Économie et Sociologies Rurales (5).

Hill J., McGowan P., Drummond P., 1999. The Development and Application of a Qualitative Approach to Researching the Marketing Networks of Small Firm Entrepreneurs. Qualitative Market Research 2 (2), 71-81.

Hite JM., 2005. Evolutionary Processes and Paths of Relationally Embedded Network Ties in Emerging Entrepreneurial Firms. Entrepreneurship Theory and Practice 29 (1), 113.

Hite J.M., Hesterly W.S., 2001. The Evolution of Firm Networks: from Emergence to Early Growth of the Firm. Strategic Management Journal 22 (3), 275.

Huault I., 2004. Une analyse des réseaux sociaux est-elle utile pour le management? Puissance et limites d'une théorie de l'encastrement structural. In Huault I. (Coord.), Institutions et Vuibert, Paris, 49-67. 
INSEE, 2011. Comprendre SIRENE. INSEE base de données SIRENE, Paris.

Jack S.L., 2005. The Role, Use and Activation of Strong and Weak Network Ties: a Qualitative Analysis. The Journal of Management Studies 42 (6), 1233-1258.

Jack S.L., Anderson A.R., 2002. The Effects of Embeddedness on the Entrepreneurial Process. Journal of Business Venturing 17 (5), 467-487.

Jaouen A., 2008. Le dirigeant de Très Petite Entreprise : Eléments typologiques, $9^{\mathrm{e}}$ CIFEPME, Louvain-laNeuve, Belgique, 28-31 octobre.

Johannisson B., 2004. Building Global Complexity and Local Diversity - Building Glocal Development Strategies. $7^{\text {th }}$ SAM/IFSAM world congress "Management in a World of Diversity and Change: Göteborg, 4-7 juillet.

Johnson J, Rasker K., 1995. The Role of Economic and Quality of Life Values in Rural Business Location. Journal of Rural Studies 11 (4), 405-416.

Jourdan N., 2004. Les transferts interrégionaux d'établissements : Forte progression entre 1996 et 2001. INSEE Première (949).

Julien P.-A., 2005. Entrepreneuriat régional et économie de la connaissance : une métaphore des romans policiers. Les Presses Universitaires du Québec, Québec.

Kalantaridis C et Bika Z., 2006. In-migrant Entrepreneurship in Rural England: beyond Local Embeddedness, Entrepreneurship and Regional Development 18, 109-131.

Kloosterman R., van der Leun J., Rath J., 1999. Mixed Embeddedness: (In) formal Economic Activities and Immigrant Businesses in the Netherlands. International Journal of Urban and Regional Research 23 (2), 252-266.

Larson L.A., Starr J.A., 1993. À Network Model of Organization Formation. Entrepreneurship Theory and Practice 17 (2), 5-15.

Morel B., Redor P., 2006. Enquêtes annuelles de recensement 2004 et 2005: La croissance démographique s'étend toujours plus loin des villes. INSEE Première (1058).

Nicot B.H. 2005. Urbain-rural : de quoi parle-t-on? Document de travail, SIRIUS, Université Paris XII Val-deMarne, Créteil, juin 2005.

North D. et Smallbone D., 2006, Developing Entrepreneurship and Enterprise in Europe's Peripheral Rural Areas: Some Issues Facing Policy-makers, European Planning Studies 14 (1).

Observatoire des territoires, 2005. Dynamique et diversité des territoires français. La Documentation Française : Paris.

Paniagua A., 2002a. Counterurbanisation and New Social Class in Rural Spain: the Environmental and Rural Dimension Revisited. Scottish Geographical Journal 118 (1), 1-18.

Paniagua A., 2002b. Urban-rural Migration, Tourism Entrepreneurs and rural restructuring in Spain. Tourism Geographies 4 (4): 349-371.

Rallet A., Torre A., 2004. Proximité et localisation. Économie Rurale 280, 25-41.

Razin E., 2002. The economic context, embeddedness and Immigrant Entrepreneurs. International Journal of Entrepreneurial Behaviour \& Research 8 (1/2), 162-167.

Reix F., 2008. L'ancrage territorial des créateurs d'entreprises aquitains : entre encastrement relationnel et attachement symbolique, Géographie, Économie, Société 10 (1), 29-41.

Reynolds P. D., 1991. Sociology and Entrepreneurship: Concepts and Contributions, Entrepreneurship: Theory \& Practice 16 (2), 47-70.

Romo F.P., Schwartz M., 1995. The Structural Embeddedness of Business Decisions: the Migration of Manufacturing Plants in New York State, 1960 to 1985. American Sociological Review 60, 874-907.

Saleilles S., 2007. L'entrepreneur néo-rural et son réseau personnel : une étude exploratoire, Thèse de doctorat en Sciences de Gestion, Université Montpellier 1.

Schutjens V., Stam E., 2003. The Evolution and Nature of Young Firm Networks: a Longitudinal Perspective. Small Business Economics 21, 115-134. 
Sencébé Y. et Lepicier D., 2007. Migrations résidentielles de l'urbain vers le rural en France : différenciation sociale des profils et ségrégation spatiale, EspacesTemps.net.

Sergot B., 2007. La personnalisation des décisions de localisation : Le cas des créations de nouveaux sites dans les secteurs de l'industrie manufacturière et de l'édition de logiciels, Finance Contrôle Stratégie 10 (4), 111-140.

Sorenson O., Audia p.G., 2000. The Social Structure of Entrepreneurial Activity: Geographic Concentration of Footwear Production in the United States 1940-1989. American Journal of Sociology 106 (2), 424-462.

Sorenson o., Stuart t., 2003. The Geography of Opportunity: Spatial Heterogeneity in Founding Rates and the Performance of Biotechnology Firms. Research Policy 32, 229-253.

Stam E., 2007. Why Butterflies Don't Leave: Locational Behavior of Entrepreneurial Firms. Economic Geography 83 (1), 27-50.

Torre A., 2009. Retour sur la notion de proximité géographique, Géographie, Économie, Société 11 (1), 63-74.

Torres O., 2003. Petitesse des entreprises et grossissement des effets de proximité. Revue Française de Gestion 144, 119-138.

Urry J., 2005. Les systèmes de la mobilité. Cahiers Internationaux de Sociologie 118, 23-35.

Uzzi B., 1997. Social Structure and Competition in Interfirm Networks: the Paradox of Embeddedness. Administrative Science Quarterly 42 (1), 35-67.

Van Dijk J., Pellenbarg P.H., 2000. Firm Relocation Decisions in The Netherlands: an Ordered Logit Approach. Papers in Regional Sciences 79, 191-219.

Williamson O. E., 1985. The Economic Institutions of Capitalism. The Free Press, London

Yin R.K., 2003. Case study Research: Design and Methods, $3^{\text {rd }} e d$. Sage, Newbury Park.

Zimmermann J.B., 1995. Dynamiques industrielles : Le paradoxe du local. In Rallet A, Torre A., Économie industrielle et économie spatiale : un état des lieux. Economica, Paris. 
